\title{
INEQUALITIES FOR A SYMMETRIC ELLIPTIC INTEGRAL ${ }^{1}$
}

\author{
B. C. CARLSON
}

AbSTRACT. Inequalities are found for an incomplete elliptic integral of the first kind which represents the reciprocal of the capacity of an ellipsoid with semiaxes $x, y, z$. One sequence of symmetric algebraic functions of $x, y, z$ converges to the value of the integral from below and two from above. Among the elements of these sequences are upper and lower approximations due to Polya and Szegö.

1. Introduction and summary. Let $x, y, z$ be positive numbers and define

$$
R=\frac{1}{2} \int_{0}^{\infty}\left[\left(t+x^{2}\right)\left(t+y^{2}\right)\left(t+z^{2}\right)\right]^{-1 / 2} d t .
$$

The electric capacity of a conducting ellipsoid with semiaxes $x, y, z$ is $1 / R[1]$. In terms of Legendre's elliptic integral $F(\phi, k)$ and the symmetric elliptic integral $R_{F}(x, y, z)[2]$, we have

$$
R=R_{F}\left(x^{2}, y^{2}, z^{2}\right)=\left(z^{2}-x^{2}\right)^{-1 / 2} F\left[\cos ^{-1} \frac{x}{z},\left(\frac{z^{2}-y^{2}}{z^{2}-x^{2}}\right)^{1 / 2}\right]
$$

It is useful for numerical and analytical purposes to approximate $R$ by an algebraic function, preferably one which, like $R$ itself, is symmetric and homogeneous of degree -1 in $x, y, z$ and has the value unity if $x=y=z=1$. Some possible candidates are

$$
\begin{aligned}
& \alpha=3 / \sum y z / x \\
& \beta=\left(3 / \sum x^{2}\right)^{1 / 2}, \quad \gamma=\left(3 / \sum x y\right)^{1 / 2}, \\
& \delta=3 / \sum(x y)^{1 / 2}, \quad \epsilon=(x y z)^{-1 / 3}, \quad \zeta=\frac{1}{3} \sum 1 / x, \\
& \eta=\left(\frac{1}{3} \sum 1 / x^{2}\right)^{1 / 2}, \quad \theta=\frac{1}{3} \sum x / y z, \quad \alpha_{1}=3 / \sum x \\
& \epsilon_{1}=\frac{2}{[(x+y)(y+z)(z+x)]^{1 / 3}}, \quad \eta_{1}=\left(\frac{4}{3} \sum \frac{1}{(x+y)(x+z)}\right)^{1 / 2}, \\
& \theta_{1}=\frac{2}{3} \sum \frac{1}{x+y} \text {, } \\
& \alpha_{2}=\frac{6}{\sum[(x+y)(x+z)]^{1 / 2}},
\end{aligned}
$$

Received by the editors November 5, 1969.

A MS Subject Classifications. Primary 3319, 6525, 4130; Secondary 7831, 2670.

Key Words and Phrases. Elliptic integrals, inequalities, ellipsoid, capacity, duplication theorem, hypergeometric $R$-functions.

1 Work performed in the Ames Laboratory of the U. S. Atomic Energy Commission. 
where $\sum$ denotes a summation over the three cyclic permutations of $x, y, z$.

We henceforth exclude the case $x=y=z$. In 1917 Pólya [3] stated the inequality

$$
\alpha<R<\theta
$$

in a problem. The solution given by Szegö [4] showed further that

$$
\alpha<\beta<R<\epsilon<\eta<\theta .
$$

Indeed, $R<\epsilon$ is a special case of Poincaré's theorem [5] that a sphere has a smaller capacity than any other conductor of the same volume. In 1945 Pólya and Szegö [1] proved a still sharper inequality,

$$
\alpha_{1}<R<\delta \text {. }
$$

It has recently been shown [6] by W. H. Greiman that

$$
\alpha_{1}<R<\epsilon_{1}
$$

and by Carlson [7] that $\epsilon_{1}<\delta$.

Let $\alpha_{n}, \cdots, \theta_{n}$ denote the result of replacing $x, y, z$ in the expressions for $\alpha, \cdots, \theta$ by $x_{n}, y_{n}, z_{n}$, where

$$
\begin{gathered}
x_{0}=x, \quad y_{0}=y, \quad z_{0}=z, \\
x_{n+1}=\left(\frac{x_{n}+y_{n}}{2} \frac{x_{n}+z_{n}}{2}\right)^{1 / 2}, \quad y_{n+1}=\left(\frac{y_{n}+z_{n}}{2} \frac{y_{n}+x_{n}}{2}\right)^{1 / 2}, \\
z_{n+1}=\left(\frac{z_{n}+x_{n}}{2} \frac{z_{n}+y_{n}}{2}\right)^{1 / 2},
\end{gathered}
$$

Thus $\alpha_{1}, \epsilon_{1}, \eta_{1}, \theta_{1}, \alpha_{2}$ have the values given in (1.3). In the present note we prove that if $n \geqq 2$

$$
\begin{aligned}
& \text { (1.9) } \quad \alpha<\beta<\alpha_{1}<\beta_{1}<\cdots<\alpha_{n}<\beta_{n}<R, \\
& \text { (1.10) } \quad R<\zeta_{n}<\eta_{n}<\theta_{n}<\cdots<\zeta_{1}<\eta_{1}<\theta_{1}<\zeta<\eta<\theta, \\
& \quad R<\gamma_{n}<\delta_{n}<\epsilon_{n}<\zeta_{n}<\eta_{n}<\cdots \\
& \text { (1.11) } \quad<\gamma_{1}<\delta_{1}<\epsilon_{1}<\zeta_{1}<\eta_{1}<\gamma<\delta<\epsilon<\zeta<\eta .
\end{aligned}
$$

These inequalities contain all the results quoted earlier, and $\beta_{n}, \zeta_{n}$, and $\gamma_{n}$ approach $R$ as $n \rightarrow \infty$. Two sequences of upper bounds are given because $\theta_{n+1}$ is not comparable with $\gamma_{n}, \delta_{n}$, or $\epsilon_{n}$. The inequalities tend to be sharp when the ratios of $x, y$, and $z$ are close to unity.

One reasonable compromise between accuracy and algebraic simplicity is $\alpha_{2}<R<\epsilon_{1}$, i.e. 


$$
\begin{aligned}
\frac{6}{\sum[(x+y)(x+z)]^{1 / 2}} & <\frac{1}{2} \int_{0}^{\infty}\left[\left(t+x^{2}\right)\left(t+y^{2}\right)\left(t+z^{2}\right)\right]^{-1 / 2} d t \\
& <\frac{2}{[(x+y)(y+z)(z+x)]^{1 / 3}} .
\end{aligned}
$$

For example, in the case $x=1, y=2, z=3, R=0.5086446 \cdots$, Equations (1.5), (1.6) and (1.12) yield

$$
\begin{aligned}
0.37<0.46 & <R<0.55<0.67<0.78 \\
0.500 & <R<0.536 \\
0.5081 & <R<0.5109 .
\end{aligned}
$$
from

Inequalities for inverse circular and hyperbolic functions follow

$$
\begin{array}{lr}
R_{F}\left(x^{2}, 1,1\right)=\left(1-x^{2}\right)^{-1 / 2} \cos ^{-1} x, & 0 \leqq x<1, \\
R_{F}\left(x^{2}, 1,1\right)=\left(x^{2}-1\right)^{-1 / 2} \cosh ^{-1} x, & x>1 .
\end{array}
$$

For example (1.12) implies

$$
\frac{6(1-x)^{1 / 2}}{2 \sqrt{ } 2+(1+x)^{1 / 2}}<\cos ^{-1} x<\frac{2^{2 / 3}(1-x)^{1 / 2}}{(1+x)^{1 / 6}}, \quad 0 \leqq x<1 .
$$

The ratio of the third member to the first increases monotonically from 1 at $x=1$ to 1.013 at $x=0$.

If exactly one of the numbers $x, y, z$ is zero, then $\epsilon, \zeta, \eta$, and $\theta$ are infinite but the inequalities between finite quantities remain valid. However, $R$ is then a complete elliptic integral for which inequalities preferable to (1.12) can readily be obtained from Gauss' algorithm of the arithmetic-geometric mean [2, Equation (5.3)], e.g.

$$
\begin{aligned}
&\left(\frac{2}{x^{1 / 2}+y^{1 / 2}}\right)^{2}<\frac{2}{\pi} R_{F}\left(x^{2}, y^{2}, 0\right)<(x y)^{-1 / 4}\left(\frac{2}{x+y}\right)^{1 / 2} \\
&(x>0, y>0, x \neq y) .
\end{aligned}
$$

Some inequalities for integrals more general than (1.1), including the capacity and surface area of ellipsoids in $n$ dimensions, are given in [8]. Some unsymmetrical nonalgebraic upper and lower bounds for (1.1) can be deduced from [9].

2. Lower bounds. We shall sharpen an inequality such as (1.5) by successive applications of the duplication theorem for elliptic integrals. This theorem has been used for iterative computation of $R$ [10], but the quantities encountered in the iteration are not completely 
symmetric in $x, y, z$. Besides the duplication theorem we shall use only two elementary results. First, the harmonic mean, the geometric mean, the arithmetic mean, and the root-mean-square form an increasing sequence. Second, Maclaurin's inequality for elementary symmetric functions states that

$$
(a b c)^{1 / 3}<\left(\frac{a b+b c+c a}{3}\right)^{1 / 2}<\frac{a+b+c}{3}
$$

provided the positive numbers $a, b, c$ are not all equal. With $a=x^{2}$, $b=y^{2}, c=z^{2}$, and $t>0,(2.1)$ implies

$$
\left(t+(x y z)^{2 / 3}\right)^{3}<\left(t+x^{2}\right)\left(t+y^{2}\right)\left(t+z^{2}\right)<\left(t+\frac{x^{2}+y^{2}+z^{2}}{3}\right)^{3},
$$

as observed in [4]. Substituting in (1.1) we have

$$
\beta<R<\epsilon,
$$

a result which follows also from [8, Theorem 2].

Continuing to exclude the case $x=y=z$, we note that $\alpha<\beta$ is implied by the identity

$$
\frac{9}{\alpha^{2}}-\frac{9}{\beta^{2}}=\sum\left(\frac{y z}{x}\right)^{2}+2 \sum x^{2}-3 \sum x^{2}=\frac{1}{2} \sum x^{2}\left(\frac{y}{z}-\frac{z}{y}\right)^{2} .
$$

Another proof, given in [4], consists in applying (2.1) to $(a, b, c)$ $=(y z / x, z x / y, x y / z)$. Furthermore the inequality of the arithmetic mean and the root-mean-square implies $\beta<\alpha_{1}$. Since $\alpha<\beta<\alpha_{1}$ implies $\alpha_{n}<\beta_{n}<\alpha_{n+1}$ by substitution of $x_{n}, y_{n}, z_{n}$ for $x, y, z$, we have

$$
\alpha<\beta<\alpha_{1}<\beta_{1}<\alpha_{2}<\beta_{2}<\cdots \text {. }
$$

The duplication theorem for elliptic integrals [10], [6] states that $R_{F}\left(x_{n}^{2}, y_{n}^{2}, z_{n}^{2}\right)$ is independent of $n$. Taken with (2.2) this implies

$$
\beta_{n}<R<\epsilon_{n}, \quad n=0,1,2, \cdots .
$$

From (2.4) and (2.5) we deduce (1.9). Moreover, $x_{n}, y_{n}$, and $z_{n}$ approach the common limit $1 / R$ as $n \rightarrow \infty$ [10], and it follows that $\beta_{n}$ and $\epsilon_{n}$ approach $R$.

3. Upper bounds. We observe first that $\gamma<\delta<\epsilon<\zeta<\eta<\theta$. The inequality of the arithmetic mean and the root-mean-square shows that $\gamma<\delta$ and $\zeta<\eta$, the inequality of the geometric and arithmetic means shows that $\delta<\epsilon$, and the inequality of the harmonic and geometric means shows that $\epsilon<\zeta$. We deduce $\eta<\theta$ from the identity 


$$
\begin{aligned}
9 \theta^{2}-9 \eta^{2} & =\sum \frac{x^{2}}{y^{2} z^{2}}+2 \sum \frac{1}{x^{2}}-3 \sum \frac{1}{x^{2}} \\
& =\frac{1}{2} \sum \frac{1}{x^{2}}\left(\frac{y}{z}-\frac{z}{y}\right)^{2}
\end{aligned}
$$

or alternatively, as in [4], by applying $(2.1)$ to $(a, b, c)=(x / y z, y / z x$, $z / x y)$.

Now $\theta_{1}$ is not comparable with $\gamma, \delta$, or $\epsilon$ because $\theta_{1}<\gamma<\delta<\epsilon$ if $x \ll y=z$ whereas $\gamma<\delta<\epsilon<\theta_{1}$ if $x=y \ll z$. However, we may conclude that $\theta_{1}<\zeta$ from the identity

$$
3 \zeta-3 \theta_{1}=\sum \frac{1}{2}\left(\frac{1}{x}+\frac{1}{y}\right)-\sum \frac{2}{x+y}=\frac{1}{2} \sum \frac{(x-y)^{2}}{x y(x+y)}
$$

or alternatively from Minkowski's inequality [11, p. 30] for the harmonic mean. Now $\theta_{1}<\zeta<\eta<\theta$ implies $\theta_{n+1}<\zeta_{n}<\eta_{n}<\theta_{n}$ and hence

$$
\cdots<\zeta_{2}<\eta_{2}<\theta_{2}<\zeta_{1}<\eta_{1}<\theta_{1}<\zeta<\eta<\theta \text {. }
$$

Since $R<\epsilon_{n}<\zeta_{n}$ by (2.5), we have proved (1.10).

To prove $\eta_{1}<\gamma$ we use the inequality of the arithmetic and geometric means to show that

$$
\left(\sum x\right)\left(\sum x y\right)>3(x y z)^{1 / 3} 3(x y z)^{2 / 3}=9 x y z
$$

and hence

$$
(x+y)(y+z)(z+x)=\left(\sum x\right)\left(\sum x y\right)-x y z>\frac{8}{9}\left(\sum x\right)\left(\sum x y\right) .
$$

It follows that

$$
\eta_{1}^{2}=\frac{8 \sum x}{3(x+y)(y+z)(z+x)}<\frac{3}{\sum x y}=\gamma^{2} .
$$

Now $\eta_{1}<\gamma<\delta<\epsilon<\zeta<\eta$ implies $\eta_{n+1}<\gamma_{n}<\delta_{n}<\epsilon_{n}<\zeta_{n}<\eta_{n}$ and hence

$$
\begin{aligned}
\cdots & <\gamma_{2}<\delta_{2}<\epsilon_{2}<\zeta_{2}<\eta_{2}<\gamma_{1}<\delta_{1}<\epsilon_{1}<\zeta_{1}<\eta_{1} \\
& <\gamma<\delta<\epsilon<\zeta<\eta .
\end{aligned}
$$

From (2.5) and (3.7) we deduce (1.11).

\section{REFERENCES}

1. G. Pólya and G. Szegö, Inequalities for the capacity of a condenser, Amer. J. Math. 67 (1945), 1-32. MR 6, 227.

2. B. C. Carlson, Normal elliptic integrals of the first and second kinds, Duke Math. J. 31 (1964), 405-419. MR 29\#1366.

3. G. Pólya, Arch. Math. und Physik (3) 26 (1917), 65. 
4. G. Szegö, Arch. Math. und Physik (3) 28 (1920), 81-82.

5. H. Poincaré, Sur un thérème de M. Liapounoff, relatif a l'équilibre d'une masse fluide, C. R. Acad. Sci. Paris 104 (1887), 622-625.

6. D. G. Zill and B. C. Carlson, Symmetric elliptic integrals of the third kind, Math. Comp. 24 (1970) 199-214.

7. B. C. Carlson, Inequality of mixed arithmetic and geometric means, SIAM Rev. (to appear).

8. - Some inequalities for hypergeometric functions, Proc. Amer. Math. Soc. 17 (1966), 32-39. MR 32 \#5935.

9. - Some series and bounds for incomplete elliptic integrals, J. Math. and Phys. 40 (1961), 125-134. MR 24 \#A259.

10. - Hidden symmetries of special functions, SIAM Rev. (to appear).

11. G. H. Hardy, J. E. Littlewood and G. P6lya, Inequalities, 2nd ed., Cambridge Univ. Press, Cambridge, 1952. MR 13, 727.

Iowa State University, Ames, Iowa 50010 